\title{
Review
}

\section{Nanoplasmonic waveguides: towards applications in integrated nanophotonic circuits}

\author{
Yurui Fang ${ }^{1,2}$ and Mengtao Sun ${ }^{1}$
}

The properties of propagating surface plasmon polaritons (SPPs) along one-dimensional metal structures have been investigated for more than 10 years and are now well understood. Because of the high confinement of electromagnetic energy, propagating SPPs have been considered to represent one of the best potential ways to construct next-generation circuits that use light to overcome the speed limit of electronics. Many basic plasmonic components have already been developed. In this review, researches on plasmonic waveguides are reviewed from the perspective of plasmonic circuits. Several circuit components are constructed to demonstrate the basic function of an optical digital circuit. In the end of this review, a prototype for an SPP-based nanochip is proposed, and the problems associated with building such plasmonic circuits are discussed. A plasmonic chip that can be practically applied is expected to become available in the near future.

Light: Science \& Applications (2015) 4, e294; doi:10.1038/Isa.2015.67; published online 5 June 2015

Keywords: nanophotonics; plasmonic chip; plasmonic circuit; surface plasmons; waveguide

\section{INTRODUCTION}

The technologies of semiconductor integrated circuits and modern electronic integrated devices are rapidly approaching their fundamental limits in terms of both speed and data transmission rate (DTR). ${ }^{1}$ One of the promising solutions to these problems is using light rather than electrons in the functional processing components. ${ }^{2}$ However, the feasibility of fabricating nanoscale photonic devices may be limited because the diffraction limit of light is dominant when the size of a photonic device is close to or smaller than the wavelength of light in the material. Surface plasmon polaritons (SPPs), electromagnetic waves coupled to charge oscillation at the metal dielectric interface, can circumvent the diffraction limit and achieve localisation of electromagnetic energy in nanoscale regions that are considerably smaller than the wavelengths of light in the material. ${ }^{3}$ The electromagnetic field perpendicular to the interface between metal and dielectric decays exponentially with distance from the metal surface while maintaining the long-range propagation of electromagnetic energy along the surface. This essential feature of SPPs can enable the fabrication of photonic components and optical signal processing devices at the sub-wavelength scale.

Surface plasmons (SPs) include the localised type (localised surface plasmons, LSPs) and propagating type (propagating surface plasmon polaritons, PSPPs). For the purpose of optical signal transportation and nanophotonic circuits, only PSPPs will be considered in this review, and they are referred to as SPPs hereafter. Because of the unique properties of tightly confined SPPs on metal structures, various types of metallic nanostructures, such as nanoparticle chains, ${ }^{4-7}$ thin metal films, ${ }^{8}$ metal slits, ${ }^{9}$ grooves, ${ }^{10}$ chemically synthesised metal nanowires $(\mathrm{NWs})^{11-13}$ and sharp metal wedges, ${ }^{14}$ have been used to guide SPPs for potential nanophotonic devices. Recently, simple plasmonic circuits composed of nanogaps on metal surfaces have been developed. ${ }^{15-17}$ In this article, we review recent development on plasmonic waveguides and plasmonic devices, mainly focus on plasmonic components related to digital circuits and try to find a way to total plasmonic chip, which is expected to have much higher speed, broader bandwidth, smaller size and lower energy consumption. The remainder of this paper is organised as follows. In Section 2, several one-dimensional plasmonic waveguides are reviewed. In Section 3, the basic components for a circuit are proposed. These include plasmonic light sources, modulators, gates, three-state gates, switches and detectors. In Section 4, a prototype of an SPP-based nanochip is presented. Finally, a way to build plasmonic chips and related problems and techniques are discussed. A summary and perspective is given at the end of this review.

\section{SPP WAVEGUIDES}

Various types of plasmonic waveguides have been proposed in recent years, including metal nanoparticle chains, metal films, metal/insulator/metal (MIM) slabs, chains of nanoparticles, NWs, metal grooves, metal strips, metal wedges, MIM gaps, hybrid Bragg waveguide and wire/spacer/film MIM structures. All of these waveguides can confine electromagnetic energy tightly near the interface, beyond the diffraction limit of light. ${ }^{18}$ In this review, we only focus on one-dimensional structures, as they are necessary for the integration of a plasmonic circuit. Figure 1 shows four types of typical plasmonic waveguides that can support the long-distance propagation of SPPs. Chemical synthesised NWs have low loss because of their single crystal structure and smooth surface (Figure 1a). ${ }^{12}$ The basic modes of wires can be

${ }^{1}$ Beijing National Laboratory for Condensed Matter Physics, Institute of Physics, Chinese Academy of Sciences, Beijing 100190, China and ${ }^{2}$ Department of Applied Physics, Chalmers University of Technology, Göteborg, SE-412 96, Sweden

Correspondence: MT Sun, Beijing National Laboratory for Condensed Matter Physics, Institute of Physics, Chinese Academy of Sciences, Beijing 100190, China

E-mail: mtsun@iphy.ac.cn

Received 29 December 2014; revised 11 February 2015; accepted 22 February 2015; accepted article preview online 24 February 2015 


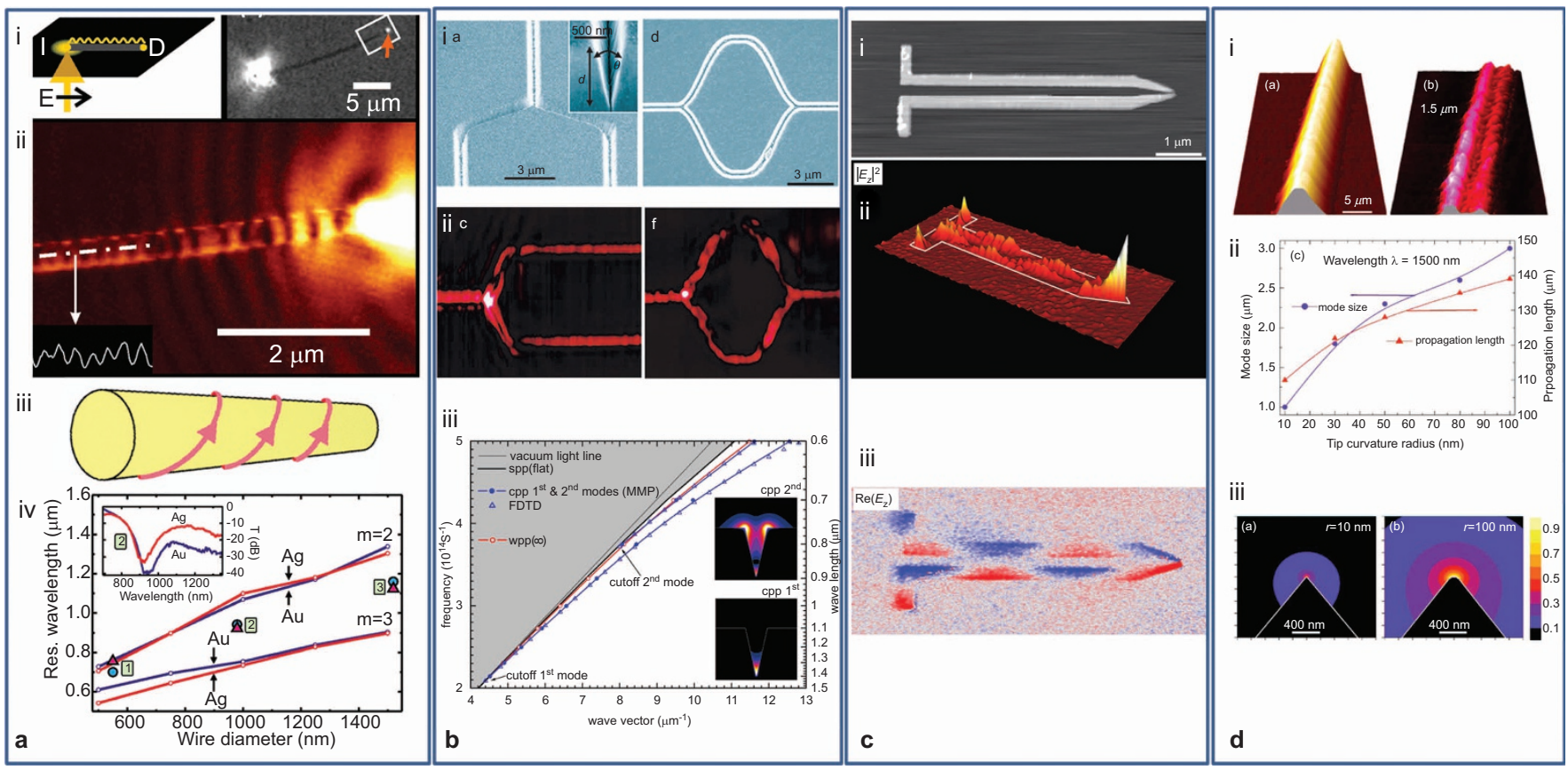

Figure 1. (a) Metal wire SPP waveguide. (i) Scheme and optical image of wire waveguide (Figure reproduced with permission: Ref. 12 @ 2005, APS); (ii) near-field image of wire waveguide; (iii) propagating mode of wire waveguide; (iv) wire radius-dependent beating period (Figure reproduced with permission: Ref. 21 @ 2008 , APS). (b) Metal groove SPP waveguide. (i) SEM images of groove waveguide (Figure reproduced with permission: Ref. 26 @ 2006, NPG); (ii) optical images of groove waveguide; (iii) the basic modes and dispersion relation of groove waveguide (Figure reproduced with permission: Ref. 24 (C) 2006, OSA). (c) Metal strips SPP waveguide. (i) SEM image of double-strip waveguide; (ii) optical image of double-strip waveguide; (iii) near-field phase shows the mode of double-strip waveguide (Figure reproduced with permission: Ref. 27 @ 2011, NPG). (d) Metal wedge SPP waveguide. (i) AFM and near-field image of wedge waveguide; (ii) the mode size and propagation length of wedge waveguide; (iii) basic mode of wedge waveguide (Figure reproduced with permission: Ref. 28 () 2008, OSA). SEM, scanning electron microscope; AFM, atomic force microscope; SPP, surface plasmon polariton.

described by the Bessel function of a cylinder, ${ }^{19}$ and the propagation of the SPPs on a metal wire can form a helix propagating beat ${ }^{20-22}$ whose period is related to the wire radius, as shown in Figure 1a(iii) and $1 \mathrm{a}$ (iv). When the wire is placed on a substrate, faster and slower modes on top and bottom of the wire appear respectively. ${ }^{23}$ For the metal groove waveguide (Figure $1 \mathrm{~b}$ ), there exists a fundamental mode and higher modes, which are called channel plasmon polariton (CPP) modes. ${ }^{24,25}$ The energy can be confined to different positions in the groove depending on the modes (wavelengths). The modes and propagation distance can be tuned through the taper angle. To effectively guide the SPPs, the depth of the groove should not be considerably smaller than the penetration depth of the fundamental mode. Figure $1 \mathrm{~b}(\mathrm{i})$ and $1 \mathrm{~b}(\mathrm{ii})$ shows a typical groove waveguide. Figure 1a(iii) shows the field distribution of basic modes and the dispersion relationships of different modes and guides. ${ }^{26}$ For the double-strip waveguide, the basic mode is similar to the lower energy mode of an MIM structure; the surface charges are opposite on two sides and oscillate along the propagation direction, as shown in Figure 1c(iii). ${ }^{27}$ The wedge waveguide is highly similar to the groove guide, but the energy is confined to the edge. The localisation can be controlled by the taper angle. ${ }^{24} \mathrm{~A}$ smaller angle will increase the localisation (Figure 1d). ${ }^{28}$ The nanoslot is highly similar to the double strips, and thus, we will not describe it further.

Not all of the one-dimensional waveguides are suitable for plasmonic circuits. The nanoparticle chains are consumed in fabrication and have high dissipative loss. The single strips are also highly dissipative because of their rough surfaces.

\section{SPP WAVEGUIDE-BASED COMPONENTS AND DEVICES}

Many devices have been developed based on the waveguide introduced above. In the following section, we explain that nearly all of the basic digital components and other necessary devices composing a complex functional circuit have already been developed. These devices include plasmonic lasers, plasmonic modulators, AND NOR gates, triple gates, control switches, routers, photon-electric converters and other useful devices, such as Mach-Zehnder Interferometers (MZIs). These plasmonic components are analogous to traditional electronic devices. The advantage is that all of the principles of electronics can be directly used to construct a plasmonic circuit without developing a new theory (e.g. quantum computation).

\section{Plasmonic laser as a nanolight source}

A light source at the nanometre scale is necessary to build a plasmonic circuit. Due to the diffraction limit of light, the coupling of the external light will occupy a considerable amount of space and will be inefficient. In addition, the size of a pure semiconductor NW-based laser device is restricted and not easy to integrate into a plasmonic waveguide circuit. ${ }^{29-31}$ Thus, to build an ultracompact laser at the nanometre scale, one strategy is to make use of surface plasmons. Xia et al. experimentally achieved plasmonic lasers that can support optical modes at the nanometre scale, and their physical size can also be considerably smaller than photonic lasers. This surface-plasmonbased nanolaser has been developed as part of the system of semiconductor NW on a metal film with a spacer supporting high optical density confined in the gap. The wires serve as Fabry-Perot resonators 

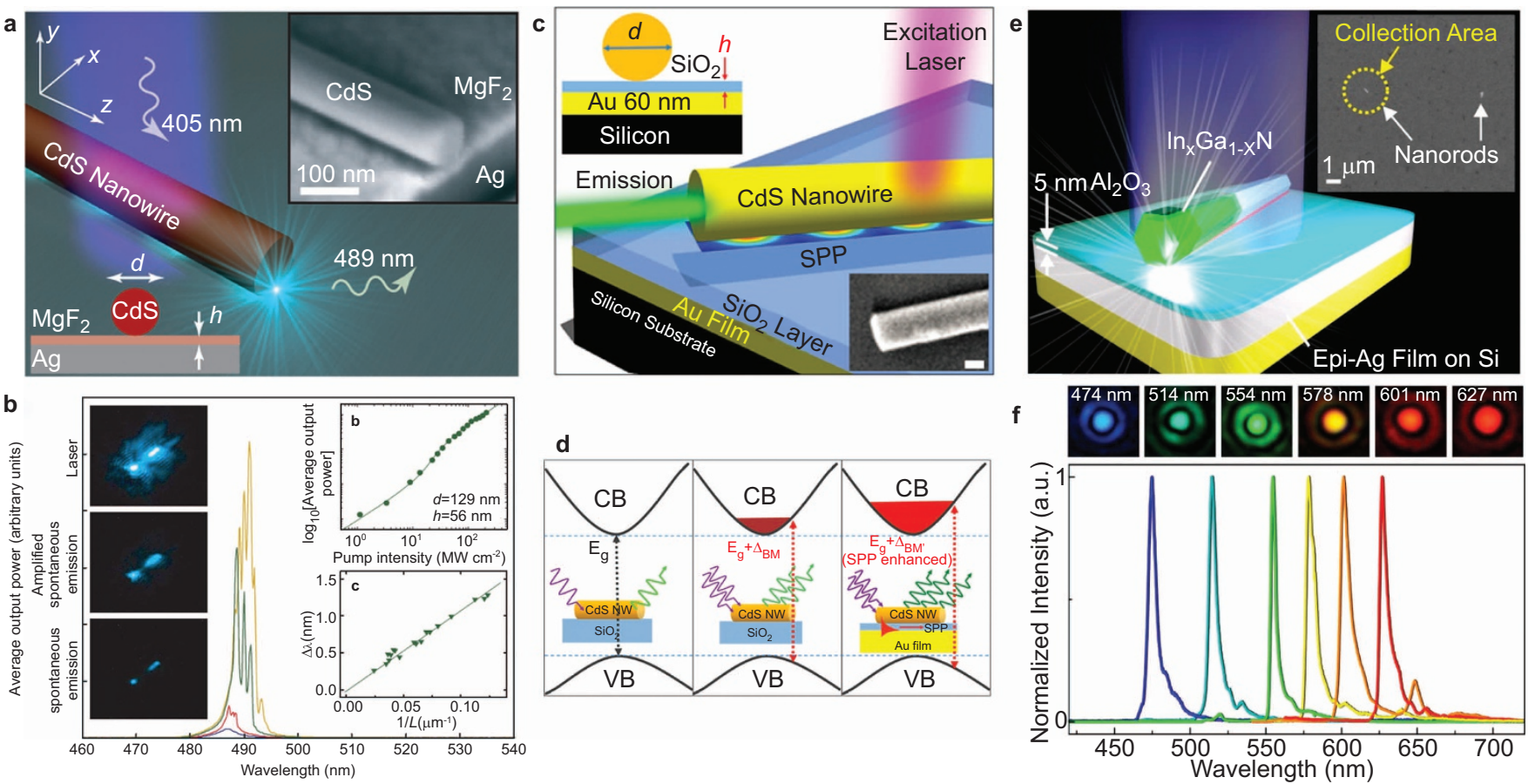

Figure 2. (a) The plasmonic laser consists of a CdS semiconductor NW on top of a silver substrate with an MgF 2 spacer. (b) Laser oscillation and threshold characteristics of plasmonic and photonic lasers (Figure 2a and 2b reproduced with permission: Ref. 32 @ 2009, NPG). (c) A schematic of the NW laser devices. (d) An illustration of the various effects on the band gap energy of non-doped semiconductor NWs (Figure 2c and 2d reproduced with permission: Ref. 33 (c) 2013, ACS). (e) All-colour InGaN/GaN nanorod plasmonic lasers. The inset shows the SEM image. (f) All-colour, single-mode lasing images observed from single nanorods with an emission line width of $4 \mathrm{~nm}$ (Figure 2e and $2 f$ reproduced with permission: Ref. 34 @ 2014, ACS). NW, nanowire; SEM, scanning electron microscope.

and optical amplification material (Figure $2 \mathrm{a}$ and $2 \mathrm{~b}$ ). ${ }^{32}$ By using the SPP-enhanced Burstein-Moss (BM) effect, the exciton emission intensities and recombination rates of the semiconductor are tuned, and thus, the emission laser wavelength can be tuned (Figure $2 \mathrm{c}$ and 2d). ${ }^{33}$ For the $\operatorname{In}_{\mathrm{x}} \mathrm{Ga}_{1-\mathrm{x}} \mathrm{N}$-based MOS laser, the emission colours can be tuned from blue to red. Thus, all colours of plasmonic lasers with a full visible spectrum were developed (Figure $2 \mathrm{e}$ and $2 \mathrm{f}$ ). ${ }^{34}$ Nanolasers based on other metal waveguide hybrid structures have been developed as well. ${ }^{35-38}$ Other light sources may be usable, ${ }^{39-41}$ but considering the wavelength-dependent properties, multiplexing and parallel data transfer with only one waveguide, nanolasers are the top priority. One of the most important (and yet to be tackled) problems is to efficiently interface a plasmonic laser and a plasmonic waveguide, whose configuration is amenable for realisation of functional components.

Electric SPP modulator as an adaptor for electric circuits and clocks The light frequency is so high that we cannot directly use it as an oscillating clock or signal input as 0 and 1 . We must integrate traditional electric components and define the strong and weak light intensities as 0 and 1 for clock and data input. Several modulators are proposed. ${ }^{42}$ Figure 3a shows an electronically controlled ultracompact SPP absorption modulator. It can be operated beyond $100 \mathrm{Gbit} \mathrm{s}^{-1}$ and is only limited by resistance-capacity time constants. It is composed of a stack of metal / insulator / metal-oxide / metal layers. It is operated by directly modulating the electron carrier density of the metal-oxide layer. ${ }^{43}$ Figure $3 \mathrm{~b}$ shows a waveguide embedded (WEB) plasmon laser, which imports $70 \%$ of the radiation into a semiconductor waveguide. This structure integrates plasmonic and electronic components, and thus, it electrically modulates and multiplexes light into the waveguide. ${ }^{44}$ Figure $3 \mathrm{c}$ shows a compact, high-speed SPP phase modulator that consists of a slot plasmonic waveguide filled with non-linear electro-optic polymer. It uses the Pockels effect of the non-linear polymer and is modulated by applying voltage on two sides of the slot. The operation speed is as high as $40 \mathrm{Gbit} \mathrm{s}^{-1}$, and it is thermally stable up to $85^{\circ} \mathrm{C} .{ }^{45}$ It is a highly suitable device for an integrated circuit clock. Considering the losses of the waveguide and compact integration, highly non-linear materials for the modulator are preferred. Other mechanisms based on nanoscale modulators can also be integrated into SPP devices. ${ }^{46,47}$

\section{SPP logic gates as basic components of digital circuits}

As core parts of plasmonic-based integrated circuits, the fundamental elementary logic gates for processing data are highly essential. Whether these logic gates can be fabricated or not will directly influence the final realisation of the nanochip. Yan et al. proposed alloptical AND OR gates based on photon-plasmon coupling in the organic-metal NW heterojunctions. The gates were fabricated through selective growth of $4,4^{\prime}$-bis-anthracene molecules on the ends of NWs. By controlling the polarisation of the incident laser, the output light at the wire end can be controlled (Figure $4 a) .{ }^{48}$ Wei et al. demonstrated the possibility of fabricating these Boolean logic gates. ${ }^{49,50}$ Branched Ag NW structures were adopted, as shown in Figure 4b. There are two input terminals, denoted I1 and I2, and one output terminal, denoted O. A laser beam was split into two beams and illuminated at I1 and I2 to excite SPP modes in NW. The phase difference between the two excitation beams can be controlled by a Babinet-Soleil Compensator. The emission intensity at the output end $\mathrm{O}$ oscillates between a maximum and minimum value when the relative phase difference increases monotonically, as shown in Figure $4 \mathrm{~b}(\mathrm{i})$. The dynamic range 

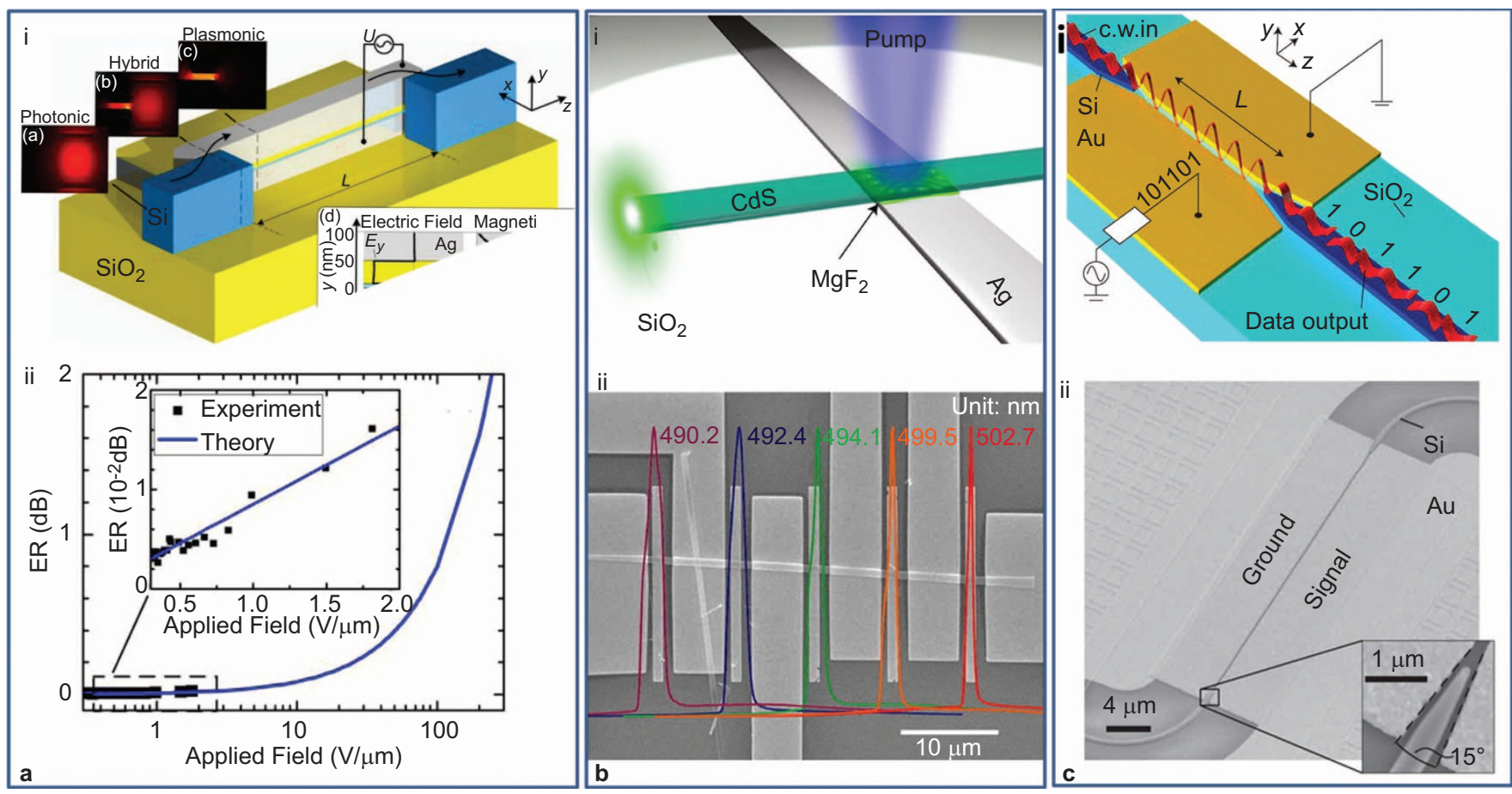

Figure 3. (a) (i) The structure of a surface plasmon polariton absorption modulator. The active section consists of a stack of silver ( $\mathrm{Ag}$ ), indium tin oxide (ITO) and $\mathrm{SiO}_{2}$ layers. The absorption coefficient of the SPP is modulated by applying a voltage between the two silver electrodes. The insets show how a photonic mode in a silicon strip waveguide excites an SPP. (ii) Predicted ER for the strong electric fields obtained from the theory using fit parameter $\omega_{p}=0.8 \times \omega_{p 0}$ and $\gamma=2.3 \times \gamma_{0}$, where $\omega_{p 0}$ and $\gamma_{0}$ are plasma and collision frequencies of ITO (Figure reproduced with permission: Ref. 43 ( ) 2011, OSA). (b) (i) Diagram of a waveguide embedded (WEB) plasmon laser that is optically pumped. (ii) WEB plasmon lasers are straightforward to assemble into integrated arrays. Here, five WEB plasmon laser devices are assembled from the same CdS strip crossing five silver strips with widths of $1 \mu \mathrm{m}$. Spectra of these five WEB plasmon lasers covering a $12 \mathrm{~nm}$ range are shown above (Figure reproduced with permission: Ref. 44 ( 2012, ACS). (c) (i) Diagram of the plasmonic phase modulator. It employs tapered silicon NW waveguides. (ii) SEM image of the plasmonic phase modulator before coating with an electro-optic polymer layer. The inset shows the coupling between the silicon NW taper and waveguide slot (Figure reproduced with permission: Ref. 45 @ 2014, NPG). ER, extinction ratio; NW, nanowire; SEM, scanning electron microscope; SPP, surface plasmon polariton; WEB, waveguide embedded.

of the ratio of maximum to minimum intensity at $\mathrm{O}$ can be greater than 10, which enables the maximum and minimum output intensities to be denoted as 'ON' and 'OFF' states, respectively. This provides a convenient way to achieve logic gates in nanophotonic circuits. Based on that work, they constructed cascaded logic gates in nanophotonic plasmon networks, as shown in Figure $4 \mathrm{~b}$ (ii). There are two branches cascaded that work as NOT and OR gates, so the function of the output end corresponds to a NOR gate. This result paves the way for the development of novel nanophotonic on-chip processors. The NOT logic gate can also be constructed by using ring resonator waveguides and modulating the radius of the rings (Figure $4 \mathrm{c}) .^{51}$

\section{SPP routers and tri-state gates as components of multiplexers and BUS}

For a pure plasmonic chip or an integrated chip with both electronic and plasmonic components, a router is necessary for a BUS with signal exchange. A tri-state gate is necessary as well because in the circuit, a third state apart from 'on' and 'off is needed. A state of 'no input' is needed so that the signals from the other components are not disturbed. In an electronic BUS, parallel data exchange requires one wire for each bit, whereas in a plasmonic wire data exchange, only one wire is needed for parallel transferring because only one plasmonic waveguide can support different wavelengths of light simultaneously and manipulate them independently, which makes it a good candidate for a router in a plasmonic circuit. It is easy to construct multiplexers and BUSs with the tri-state gate and router. Kim et al. principally constructed a switch that can be used as a tristate gate. ${ }^{52,53}$ By tuning and changing the number of equal transition frequencies of quantum dots coupled to one-dimensional SPP waveguide, the transmission and reflection of a single plasmon can be switched on or off (Figure 5a). Chang et al. also developed a single photon transistor that can act as a non-linear two-photon switch for the SPPs propagating on an NW. The propagation of the SPPs on a wire waveguide can be altered by the interaction of the SPP and single two-level emitter. For lower power, because the SPPs excite the emitters and cause it to reemit, there is a phase difference, and thus, the SPP can be reflected with high efficiency (Figure $5 b(i)) .{ }^{54}$ Thus, the single emitter behaves as a saturable mirror because it cannot scatter more than one photon at one time. An ideal single phototransistor can be developed through a multilevel emitter (Figure 5b(ii)). Using this principle, a single photon tri-state gate is developed. In addition, a nanoplasmonic router was designed by Fang, as shown in Figure $5 c{ }^{55}$ For a branched wire structure, using lasers to illuminate one end of the wire, through varying the illuminating polarisation, the light can be controlled to emit from end 2 or 3 . Moreover, if two lasers with different wavelengths, 633 and $785 \mathrm{~nm}$, were focused at the same end of the structure, then rotating the polariser, a $633 \mathrm{~nm}$ laser will come out mainly from end 2 and a $785 \mathrm{~nm}$ laser will come out mainly from end 3 . Such routers supporting multi-wavelength modes meet the requirement of transferring data in parallel with only one wire. Wavelength selective plasmonic multiplexer utilising CPPs 

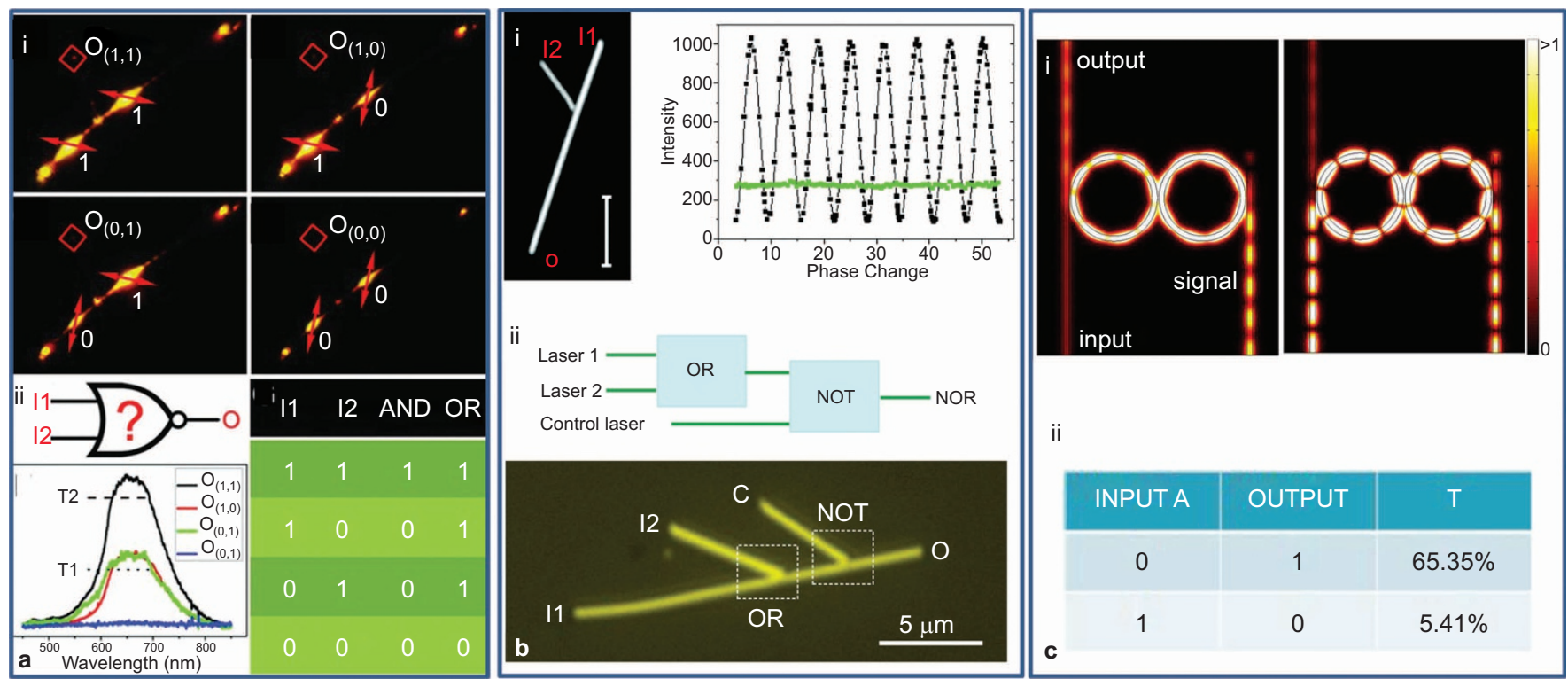

Figure 4. (a) Realisation of the photonic logic processing. (i) PL microscopy images under the excitations of four different combinations of the two polarised inputs. Red arrows indicate the polarisation directions of the two laser beams. Here, 1 and 0 were defined according to the intensities of the scattered SPPs. (ii) The basic logic gate built with two simultaneous inputs and one output signal, summary of optical logic operations for AND and OR gates and the scattering spectra of SPPs at the O-terminal under the excitations shown in (i). T1 and T2 represent the thresholds for the OR and AND gates, respectively (Figure reproduced with permission: Ref. 48 ๑ 2012 , WILEY-VCH Verlag GmbH \& Co. KGaA). (b) Branched NW as an optical logic gate. (i) Optical image and scattering intensity at output end $\mathrm{O}$ as a function of phase delay between two incident beams (Figure reproduced with permission: Ref. 49 @ (2011, NPG). (ii) Diagram of a NOR logic gate built using cascaded OR and NOT gates (top) and optical image of designed Ag NW structure (bottom) (Figure reproduced with permission: Ref. 50 @ 2011, ACS). (c) A plasmonic NOT gate structure. (i) The magnetic field distribution. (ii) The truth table of the NOT gate ${ }^{51}$ (reproduced courtesy of The Electromagnetics Academy). PL, photoluminescence; SPP, surface plasmon polariton.

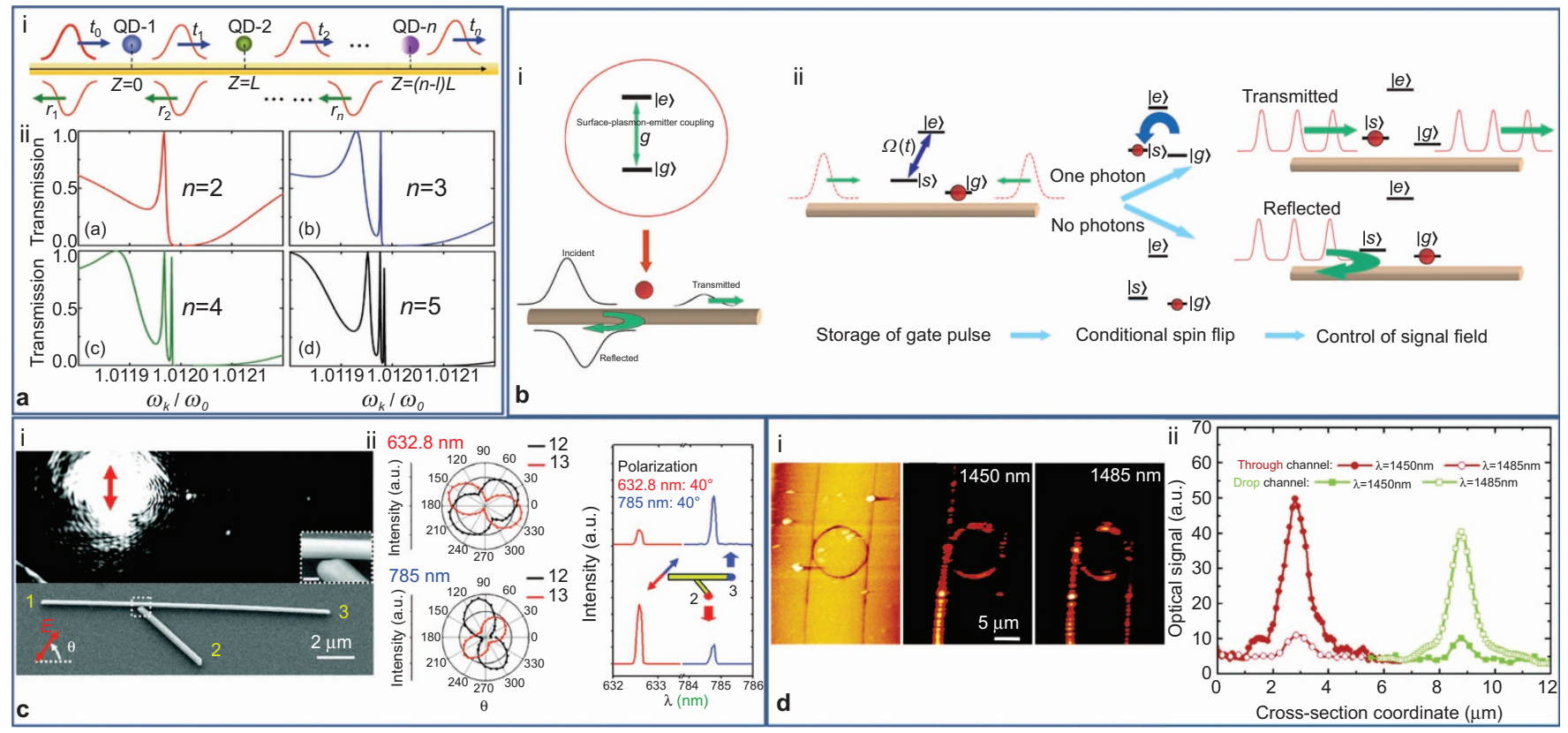

Figure 5. (a) (i) Diagram of a system consisting of a single plasmon and $n$ equally spaced QDs coupled to a metal NW. $t_{\mathrm{i}}$ and $r_{\mathrm{i}}$ are the transmission and reflection amplitudes at the place $z_{i}$, respectively. (ii) Transmission spectra of a propagating plasmon interacting with $n Q D s\left(\Omega i=1.0125 \omega_{0}, i=1,2, \ldots, n\right)$ versus $\omega_{k}$ for phases $k_{\mathrm{L}}=0.1 \pi$, where the frequency is in units of $\omega_{0} \equiv 2 \pi v_{\mathrm{g}} / \mathrm{L}$ and $J=10^{-4} \omega_{0}$ (Figure reproduced with permission: Ref. 53 () 2014, Springer). (b) (i) Two-level emitter interacting with the NW. States $|g\rangle$ and $|e\rangle$ are coupled via the surface-plasmon modes with a strength $g$. The interaction leads to reflected and transmitted light of a single SPP on the wire. (ii) A gate pulse consisting of zero or one photon is split equally in counter-propagating directions and coherently stored using an impedancematched control field $\Omega(t)$. The storage results in a spin flip conditioned on the photon number. A subsequent incident signal field is either transmitted or reflected depending on the photon number of the gate pulse, owing to the sensitivity of the propagation to the internal state of the emitter (Figure reproduced with permission: Ref. 54 ( 2007, NPG). (c) An Ag nanobranch waveguide router. (i) Optical image of the nanobranch excited by a laser (top) and corresponding SEM image (bottom). (ii) The spectra collected from wire end 3 (top) and 2 (bottom) when the incident polarisation angle is $40^{\circ}$ (Figure reproduced with permission: Ref. 55 @ 2010 , ACS). (d) (i) Pseudocolor topographical image, and near-field optical images at $1450 \mathrm{~nm}$ and $1485 \mathrm{~nm}$. (ii) Normalised cross sections obtained from near-field images (Figure reproduced with permission: Ref. 56 @ 2007, ACS). NW, nanowire; QD, quantum dot; SEM, scanning electron microscope; SPP, surface plasmon polariton. 
with milled waveguide-ring resonator grooves has been realised earlier as well (Figure $5 \mathrm{~d}) .{ }^{56}$

\section{SPP plasmonic MZI as a switch and adaptor for gates}

To complete an optical nanochip, a switch at the nanometre scale is essential for enabling or disabling the function of a component or turning the signal on and off. Conventional optical switches are excluded due to their large physical size, which makes them incompatible with the nanochip. A traditional complementary metal-oxide-semiconductor transistor transistor-based electronic switch is too slow for the plasmonic circuit, which makes the circuit similar to traditional electronics. Thus, a new type of switch is required. Based on a local non-linear MIM plasmonic MZI waveguides, $\mathrm{Wu}$ et al. proposed an all optical switch (Figure 6a). ${ }^{57}$ The local Kerr-type non-linear MIM plasmonic MZI waveguide is used in this switching structure because of its large thirdorder non-linear susceptibility and ultrafast response properties. By properly fixing the input signal power and changing the control power, the output signal beam will exhibit the switching effect. Ming et al. proposed a resonance coupling-based plasmonic switch. It is composed of a single gold nanorod and a surrounding photochromic dye (Figure 6b).$^{58}$ The entire component is less than $100 \mathrm{~nm}$. It is controlled by ultraviolet light. The modulation depth of the plasmonic switch reaches $7.2 \mathrm{~dB}$. The estimated laser power and energy required for operating a single-nanorod plasmonic switch are $13 \mathrm{pW}$ and $39 \mathrm{pJ}$, respectively. Other plasmonic switches have also been developed to interfere with PSPPs based on phase controlling. ${ }^{59}$

In addition to constructing a switch, MZI is also critical in plasmonic devices. It is useful and self-stable in the circuit, many important parts have phase and polarisation interfering principles. The plasmonic logic gates are highly sensitive to the wire connection position because of the phase delays on the wire. This is different to simple research in the laboratory, where the input light phase or position can be well controlled; in large-scale fabrication, small mistakes can cause total failure of the entire circuit. With an MZI, the phase of the connection can be fine-tuned in some components. Such hybrids MZIs composed of dielectric fibres and metal NWs or pure metal NWs have been proposed for sensing applications, ${ }^{60,61}$ and they are highly useful in plasmonic circuits. The fringes of a free space range (FSR) can be theoretically calculated by the following equation:

$$
\mathrm{FSR}=\lambda^{2} /\left(L_{1} n_{1}-L_{2} n_{2}\right)
$$

As illustrated in Figure $6 \mathrm{c}(\mathrm{i})$, due to the favourable wave-guiding properties of plasmonic NW waveguides, e.g., tight confinement and field enhancement, the NW MZI offers great potential for ultracompact functional components and optical sensors (Figure 6c). The waveguiding properties of Au NWs for plasmonic sensing in liquids are different; thus, the plasmonic MZI can be used to measure the phase shift introduced by the index changes of the surroundings.

All optical logic discriminators were also developed by adopting the plasmonic bandgap engineering (Figure 6d). The nanoslit and left plasmonic crystal constructed a wideband and unidirectional SPP light source. The right plasmonic crystal performed the function of wavelength discrimination. The SPPs are reflected or pass through the plasmonic crystal according to their wavelengths, which represent a logic state of ' 0 ' or ' 1 '. This is useful in the signal routing in plasmonic BUS. In addition, the wavelength-selective properties of plasmonic waveguides and other waveguides are also usable in the BUS system. ${ }^{62,63}$

\section{SPP waveguide detector as an interface to an electric circuit}

The principle of the plasmonic circuit is consistent with traditional electronics, and the two types must be integrated together to adapt existing electronic devices. Thus, it is important to convert the plasmonic signal to electric signals. Metallic NW plasmonic waveguides offer opportunities to carry optical signals and electric currents through the same metal circuitry, and thus, they have the ability to merge electronics and photonics on the same chip, where they can take advantage of the advanced electronic integrated technology and the
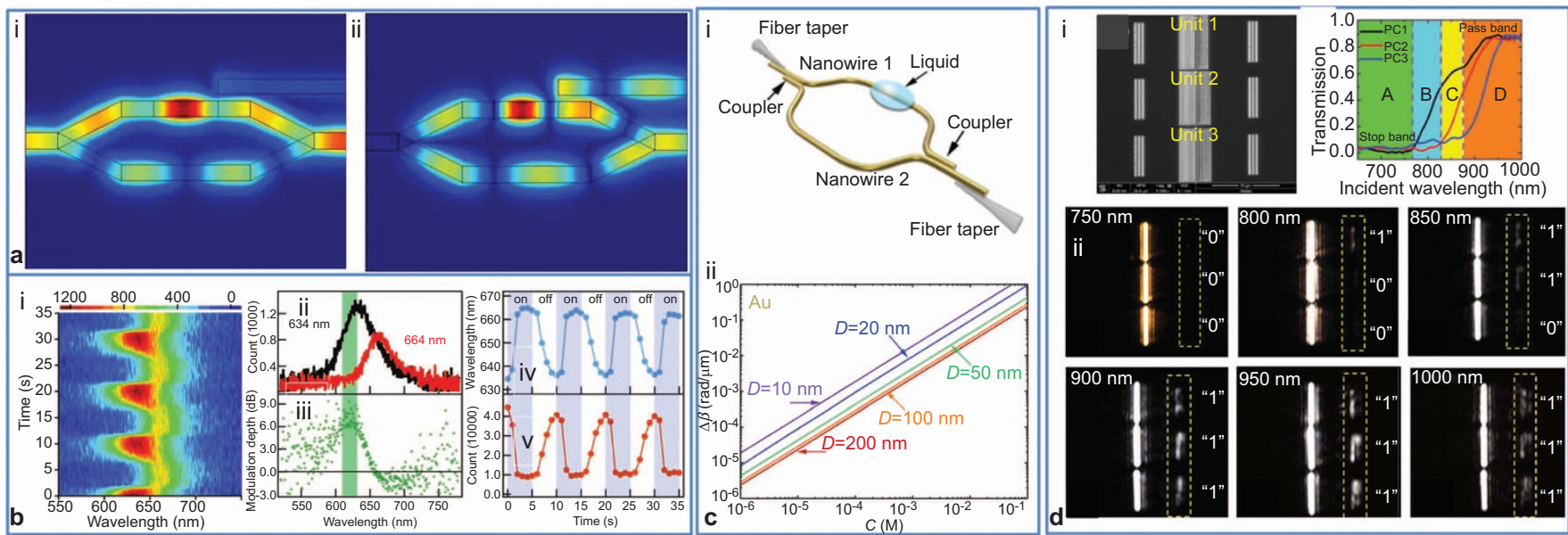

Figure 6. (a) Magnetic field distributions of signal light with switching (i) off and (ii) on. ${ }^{57}$ (Figure reproduced courtesy of The Electromagnetics Academy) (b) Switching dynamics of a representative Au nanorod. (i) Contour plot showing the evolution of the scattering spectra. (ii) Scattering spectra of the Au nanorod acquired when the ultraviolet laser illumination is off (black) and on (red). (iii) Scattering intensity modulation depth versus wavelength. (iv) Variation of the scattering peak wavelength with time. (v) Variation of the scattering intensity with time. The scattering intensity is integrated from $610 \mathrm{~nm}$ to $630 \mathrm{~nm}$, as indicated by the green shaded region in (iv) and (v) (Figure reproduced with permission: Ref. 58 @ 2010, WILEY-VCH Verlag GmbH \& Co. KGaA). (c) (i) Diagram of a proposed sensor with an $\mathrm{MZI}$. (ii) Dependence of the change in the propagation constant $(\Delta \beta)$ of Au NWs on the molar concentration of benzene $(C)$ in aqueous solution, with nanowire diameters of 10, 20, 50, 100 and $200 \mathrm{~nm}$. The wavelength of light used here is $660 \mathrm{~nm}$ (Figure reproduced with permission: Ref. 61 ( ) 2014, IEEE). (d) All of the optical logic discriminators (Figure reproduced with permission: Ref. 17 @ 2014, Springer). 
high speed, and high bandwidth of photonic circuits. Therefore, the integration of plasmonic waveguides with electrical circuits is of great significance. Falk et al. reported an electrical SPP detection technique based on the near-field coupling between guided plasmons and an NW field-effect transistor. ${ }^{64}$ The experimental schematic is shown in Figure 7a. The propagating SPP modes in Ag NW are coupled to nearby Ge NW detectors. By scanning a focused laser beam across a Ag-Ge crossbar device, the current (I) through the Ge NW as a function of the laser spot position was recorded. The ratio of detected charges to the number of SPPs reaching the Ag-Ge junction is defined as the plasmon-to-charge conversion efficiency $(\eta)$, which is approximately 0.1 electrons per plasmon. This efficiency can be improved to approximately 50 electrons per plasmon using a plasmonic gating effect, where the Ag NW works as both the plasmonic waveguide and an electrical gate for the Ge NW. This device is in nanoscale and demonstrates the possibility of future on-chip optoelectronic devices. The detector was also built as an MIM waveguide combined with a fast slit metal-semiconductor-metal photodetector (Figure 7b). ${ }^{65}$ Goykhman et al. even proposed a more suitable on-chip silicon surface plasmon Schottky detector based on the groove waveguide structure (Figure 7c). ${ }^{66}$ The advantage of this detector is that it can be easily integrated with the other components with the traditional fabrication technique. They realised efficiency of 0.25 and $13.3 \mathrm{~mA} \mathrm{~W}^{-1}$ at the wavelengths of 1.55 and $1.31 \mu \mathrm{m}$, which are the working wavelengths of modern fibre communication.

\section{TOWARDS A PLASMONIC CIRCUIT}

\section{Prototype for an SPP waveguide-based nanochip}

Based on plasmonic waveguides, we have reviewed plasmon nanolasers, nanomodulators, plasmonic gates, plasmonic tri-state gates, routers, plasmonic switches, plasmonic repeaters and plasmonic detectors. The research on these devices indicates that we can nearly construct an entire plasmonic circuit. Here, we refer the entire plasmonic circuit not only as a simple, basic component, but an integrated chip with complete function, such as a D/A convertor, a comparer, an integrated operational amplifier, or even a single-chip microcomputer (SCM) with a central processing unit (CPU). We now follow the traditional electronic circuit ${ }^{67}$ to construct the plasmonic circuit but not to develop a total new circuit, such as quantum computation. In such a circuit or CPU, all of the binary mathematics and principles can be used directly, and the components are sufficient to construct a CPU in principal (Figure 8). According to principle of the electronic digital chips, a simple arithmetic logic unit (ALU) chip (e.g. SN74181) with simple arithmetic and logic function can be constructed with the basic AND, OR, NOT gates. Several simple ALU chips and multiplexer can be used to construct a powerful ALU. Registers are composed of D flip-flops, which can be constructed with the three basic gates as well. A complex CPU thus can be constructed with very simple components. The speed of such a chip is still limited by the electronic-plasmon switch. However, by replacing all of the basic components with plasmonic ones, electronic switching will be significantly reduced with only input and output connection left. Thus, the processing speed is increased by thousands of times.

\section{Barriers and outlook}

Simple integrated SPP waveguide-based circuits have already been described recently. Burgoes et al. proposed a complex router of plasmon v groove guide networks (Figure 9a). Huang et al. described an electrically driven nanoscale light-emitting diode (LED) that can work as a nanoscale plasmonic source to excite plasmon in the metallic waveguide through gap plasmon mode as shown in Figure 9b. ${ }^{16}$ They also demonstrated a nanoscale T-splitter using this source. These results facilitate the integration of electrically driven sources and plasmonic circuits such as routers, with splitting at the sub-wavelength scale. A routing and detection of SPPs on slot waveguide has also been fabricated by Ly-Gagnon et al. ${ }^{68}$

The fundamental functional components for a CPU chip are almost available now, but the applicable nanophotonic circuits are still far from what is desired-a real integrated device. The common fastresponse electronic devices are typically on the nanosecond time scale. Recently, researchers have developed a $100 \mathrm{GHz}$ detector based on graphene electronics. ${ }^{69}$ However, the integrated complex electronics will still be $\mathrm{GHz}$ speed because many switches decay the response in each individual component. Other methods, such as quantum computation, are considered to be the best way to overcome this barrier. However, there are many problems that need to be solved, and those problems are more difficult than those associated with plasmonic devices. As we shall see in the review, all of the basic components are there, and they can be used in simple ways-only a small number of waveguides is needed to build a functional device, although the electronics are more complex. The simple structure of the plasmonic device will make the device more compact, and the number of basic functional components will be greatly reduced. Thus, the time delay only comes from the input, output and SPPs propagating time along the guides. Although there are electronic switches in the input and output parts, the time scale of the entire processing unit (but not each
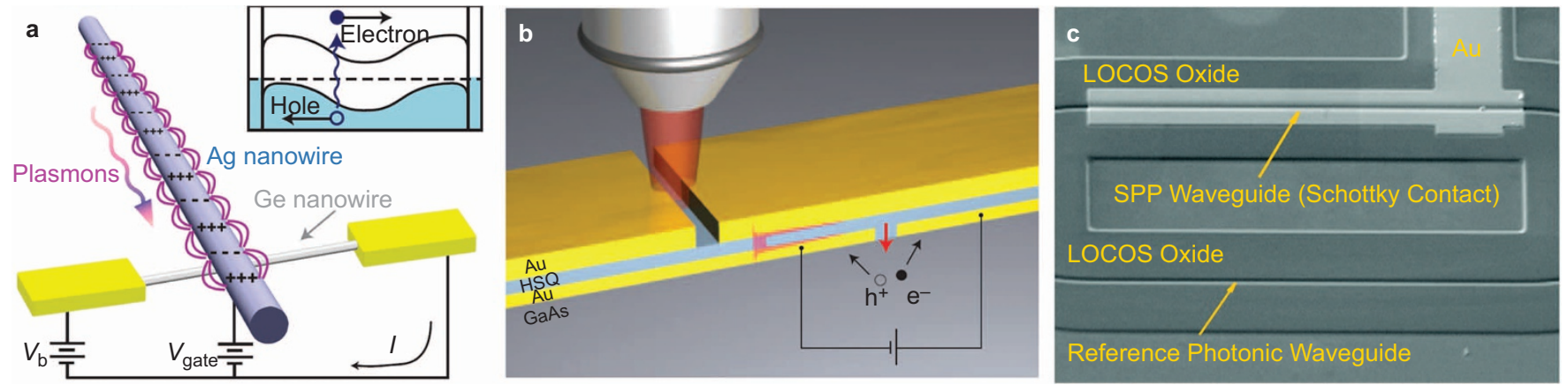

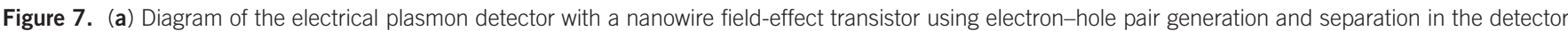

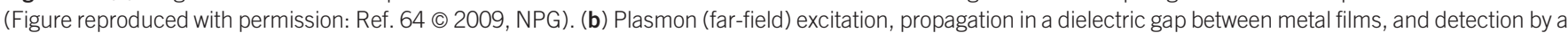

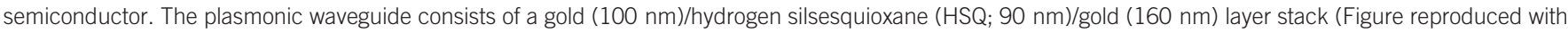

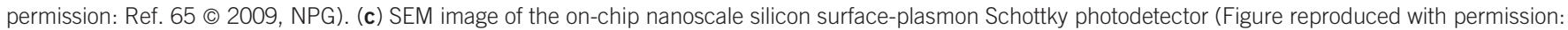
Ref. 66 (c) 2011, ACS). SEM, scanning electron microscope. 


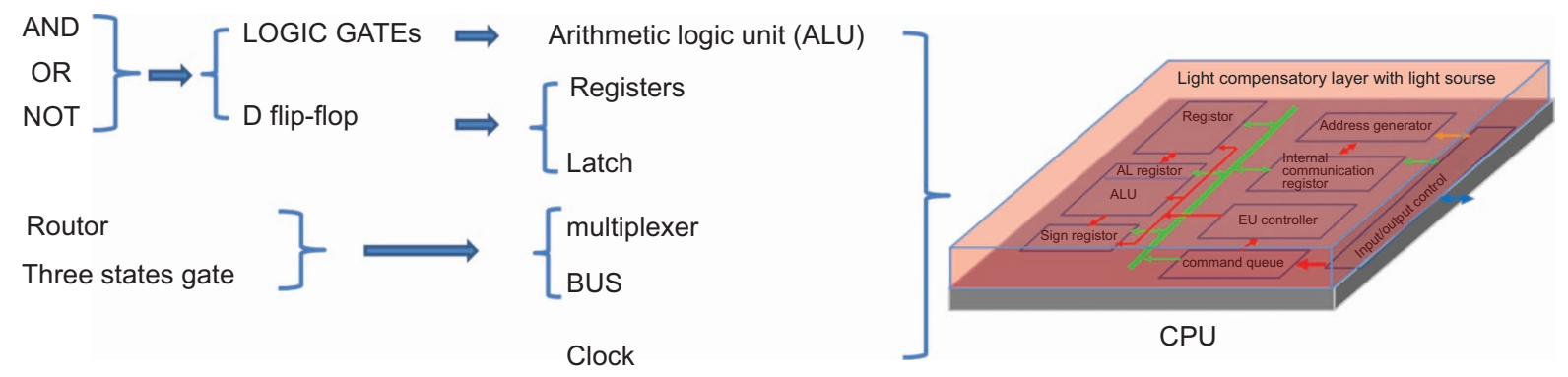

Figure 8. Prototype of a plasmonic circuit. Each functional part can be built from one or more previously described components.

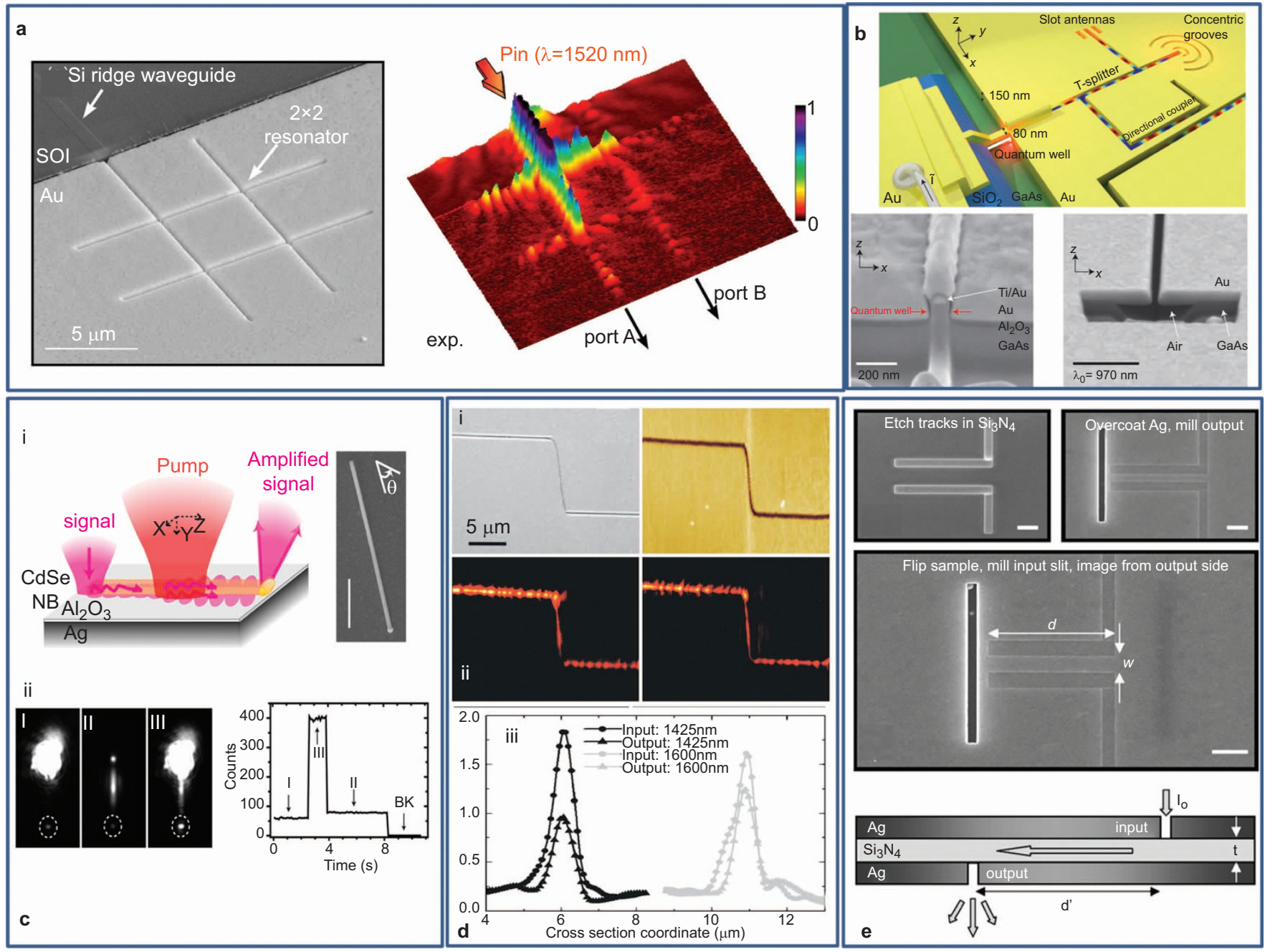

Figure 9. (a) Plasmonic circuit made of nanogaps (Figure reproduced with permission: Ref. 15 @ 2014, ACS). (b) Plasmonic circuit made of nanogaps with quantum wells as a light source (Figure reproduced with permission: Ref. 16 (c) 2014, NPG). (c) (i) Diagrams of the CdSe NB/Al $\mathrm{O}_{3} / \mathrm{Ag}$ hybrid plasmonic waveguides in compensation mode as a plasmonic repeater and SEM image of one structure. (ii) Optical images corresponding to a probe signal of photoluminescence (PL) with or without the pump, and time trace of the output intensity at the emission end marked with dashed circles on the left (Figure reproduced with permission: Ref. 71 ( 2013, NPG). (d) (i) SEM image with topographical and (ii) near-field optical images $\left(26 \times 21 \mu \mathrm{m}^{2}\right)$ taken at $\lambda \cong 1425$ and $1600 \mathrm{~nm}$. (iii) Cross sections obtained with the optical images shown in (ii) by using 3- $\mu \mathrm{m}$-long averaging windows along straight groove regions before and after the $S$ bend (Figure reproduced with permission: Ref. 74 ( 2006, AIP). (e) Slot waveguide fabrication sequence and experimental set-up. Ag is evaporated on one side of a $\mathrm{Si}_{3} \mathrm{~N}_{4}$ membrane before focused ion-beam milling is used to define the waveguide core (the nitride remaining between the tracks). The exposed nitride is then coated with $\mathrm{Ag}$, and the output slit is milled. The sample is flipped, and the input slit is milled (Figure reproduced with permission: Ref. 80 ๑ 2006, ACS). SEM, scanning electron microscope. 
step) would depend on the speed of the electronic-plasmonic switch. The delay from the plasmonic waveguide is negligible, as the propagation speed is still in the magnitude of light speed. Thus, the future of plasmonic devices is bright, and their realisation in near.

In such a plasmonic circuit, the bandwidth will be extremely increased. Usually for a plasmonic waveguide, it works in a very broad wavelength range, and lasers are used to communicate. Even though some router components are wavelength dependent, the routable wavelengths in a small range for the same route by one router are still possible in the threshold level. Considering the narrow width of the laser line, the bandwidth would be very large for even one waveguide. If several wavelength ranges are operating synergistically (parallel data transmission with only one guide), the bandwidth would be considerably large only with one waveguide. The evaluation of the plasmonic chip bandwidth is comparable to the optical silicon chip, which is done by Miller. ${ }^{70}$

Realisation of the tremendous potential of SPP-based nanophotonic circuits will depend on resolving several serious issues, such as the implementation of functional components within the limitations imposed by inevitable SPP propagation losses, and efficient coupling between nanoscale waveguides.

\section{Energy loss and power compensation}

Energy attenuation is always a problem, in both electronic and plasmonic circuits. Ditlbacher and co-workers investigated surface plasmon modes in chemically prepared $\mathrm{Ag} \mathrm{NW}$ and found that the scattering loss is practically eliminated with these modes on monocrystalline NWs, resulting in unexpectedly large propagation lengths. ${ }^{12}$ The propagation loss can be described by the surface plasmon propagation length over which the intensity of the SPP decreases to $1 /$ e of the initial value. In some studies, the propagation length can reach $1 \mathrm{~mm}$. The propagation of SPPs on metal waveguides is also affected by other factors, such as the spacer and dielectric medium in the gaps. However, the attenuation arising from absorption in the metal will be significant if the length of the guide is tens of kilometres in total anagogic to electronic chips. Therefore, to design SPP-based devices, it is important to relay the signals. Even in the simplest chips, a power supply is needed.

Compared with the gate voltage and current supply in the transistors (converting signal between current and voltage) in electronic chips, the signal processing in a plasmonic circuit can be integrated with a repeater and switch, as only propagated light intensity and polarisation is needed for the signal. Such a repeater was developed simply by using quantum dots as a signal amplifying material and by shining excitation light on the wire waveguide. The developed repeater can maintain the properties of the input signal. Figure $9 \mathrm{c}$ shows that $\mathrm{CdSe}$ nanobelt $/ \mathrm{Al}_{2} \mathrm{O}_{3} / \mathrm{Ag}$ hybrid plasmonic waveguides allow for efficient broadband loss compensation of propagating hybrid plasmonic signals of different polarisations using an optical pump and probe technique. ${ }^{71}$ With an internal gain coefficient of $6755 \mathrm{~cm}^{-1}$ under ambient conditions, nearly $100 \%$ of the propagation loss of TM-dominant plasmonic signals is compensated. Based on this property, a plasmonic repeater for amplifying the signals during propagation is developed. The amplification of long-range surface plasmons on a symmetric metal stripe by a dipolar gain medium has also been realised by Leon et al. The measure mode power gain is $8.55 \mathrm{~dB} \mathrm{~mm}{ }^{-1} .^{72}$ The repeater can be fabricated in such a way: first isolate the connections of the waveguides with other dielectric material, and then put signal-amplifying material in the middle part of the waveguides. Thus, excitation light can be shined on the entire device. Such light compensation devices in groove waveguide is also very necessary in the future integration, and need further research.

Energy attenuation from the bending part of the guide is also extremely important. It is the most lossy part aside from the far field emission of the junctions. Wang's study shows that the loss is dependent on the radius of the bending. ${ }^{73}$ However, in the groove guide, after two bends, the signal is still sufficiently strong for detection (Figure 9d). ${ }^{74}$

In the integrated complex plasmonic circuit, the main energy consumption comes from the scattering of the junction, the heating loss, and the amplification materials for the light sources. One cannot avoid the heating loss, but compared with the scattering of the junctions, the other losses are very little. A total reflective dielectric cover on the junction can considerably reduce the energy waste. Even though, compared with the electronic chips, the total energy consumption of plasmonic chip is expected much lower, because all of the operations are just SPs propagating and interference (most of the connection and functional parts are gold, which has high conductivity). One topic to be addressed in the near future is single-plasmon ${ }^{75}$ switching and routing, which may make the devices even simpler and lower energy consumption, and the light compensation may not be necessary.

\section{Integration of different components}

If one constructs a complex plasmonic device, e.g., a simple ALU with just basic functionality, the units reviewed above are in principal already enough. However, it is difficult to arrange them correctly. These components are fabricated in a laboratory individually, and most of them are controlled by external methods. There are components for phase delay, polarisation control and coupling position control through an external tool, such as a microscope or scanning near-field optical microscope (SNOM), with other macro-optical components, such as polarisers. In particular, the polarisation-based components are defined under the microscope, and the polarisation is measured on the plane parallel to the substrate. ${ }^{76}$ It will be different at the junction and when measured from the next components if they are integrated. The functions of these components are mainly unknown before the measurements. When the components are integrated together on a chip, the polarisation-controlled ones are not suitable, even though there are some declared nanopolarisers. ${ }^{77}$ Considering the integration, the intensity is currently the best way (based on the principle introduced above) to define the signals ' 0 ' and ' 1 '. The components are based on the interference of SPPs and controlled by the phase and excitation of different basic modes of the SPP waveguides. The phase (to realise the correct function) can be tuned by using an MZI with external material on one arm. The excitation of different modes can be controlled through excitation wavelengths and exciting positions (which are related to the fabrication). However, those components introduced above mainly work at specific wavelengths. ${ }^{58} \mathrm{How}$ to make them compatible with several fixed wavelengths remains an unresolved issue.

Table 1 Comparison of different plasmonic waveguides for a circuit.

\begin{tabular}{|c|c|c|c|c|c|c|}
\hline Waveguide type & Chemical synthesised wire & Slot (MIM hybrid strips) & Strip & V-shaped metal dielectric groove & Wedge & Particle chain \\
\hline Energy loss & Low & Middle & High & Middle & Middle & Very high \\
\hline Fabrication for integration & Very hard & Easy & Easy & Easy & Harder & Hard \\
\hline
\end{tabular}




\section{Fabrication}

This is the most difficult part: fabrication. The components introduced above are based on different SPP waveguides: NWs, grooves, wedges, slots, strips, slabs, MIMs, wire-film hybrid structures and more. In an integration procedure, it is not possible to put all of the different waveguides together; it is better to use one technique. Chemically synthesised wire components are fabricated through micro-manipulation, but it is impossible to use such a tool to make an integrated chip to manipulate kilometres of wires one by one. This will remain impossible unless some technique can grow single crystal metal wires on designed pattern on a substrate. The other waveguides are suitable for integration fabrication. Microfabricated nanostrips have rough surfaces and scatter a significant amount of light. It is possible to fabricate ultra-smooth wedges without using chemically synthesised single crystal particles, ${ }^{78,79}$ but the processing is extremely complex. Currently, the grooves and slots are good candidates. MIM layers are useful as well because multilayer component structures (such as electronic chips) cannot be completely avoided (Figure 9e).$^{80}$ This is also another way to amplify the signals by putting optical amplification materials between metal layers and shining light through the MIM guide. It is also easier to fabricate high-quality groove or slot waveguides because one can obtain ultra-smooth grooves or slots by etching a single crystal silicon substrate and evaporating high-quality films. ${ }^{78,79,81-83}$ In such whole groove or slot integration, the hybrid wire/spacer/film laser is still usable, as it does not require many laser sources. Some advantages and disadvantages of different SPP waveguides are listed in Table 1. In addition, circuits based on full grooves or slots require redesigning and testing certain components. For example, they require replacing the NW-based repeater with a groove-based repeater.

\section{SUMMARY}

This paper provides a comprehensive overview of the basic mechanism and propagating properties of PSPPs, and it describes recent progress in applicable SPP-based nanophotonic components and devices. The fundamental properties are nearly clear, and the applicable nanophotonic circuits are further developed but still far from what are desired-a real integrated device that can be used in actual optoelectronic devices. Because the basic components (NW-based laser, BUS router, switch, adder, NAND gate) are already available, the future of nanophotonics is bright. One topic to be addressed in the near future is single-photon switching and routing. Single photons do not 'talk' to each other, but efforts are under way to use plasmonmediated interactions for this purpose. It is also possible that hybrid excitation that combines photons and electrons will be the carrier of future quantum information systems. ${ }^{84}$

\section{ACKNOWLEDGEMENT}

This work was supported by National Nature Science Foundation of China (Grant Nos. 91436102, 11374353 and 11474141).

1 Gramotnev DK, Bozhevolnyi SI. Plasmonics beyond the diffraction limit. Nat Photonics 2010; 4: 83-91.

2 Zia R, Schuller JA, Chandran A, Brongersma ML. Plasmonics: the next chip-scale technology. Mater Today 2006; 9: 20-27.

3 Barnes WL, Dereux A, Ebbesen TW. Surface plasmon subwavelength optics. Nature 2003; 424: 824-830.

4 Maier SA, Kik PG, Atwater HA, Meltzer S, Harel E et al. Local detection of electromagnetic energy transport below the diffraction limit in metal nanoparticle plasmon waveguides. Nat Mater 2003; 2: 229-232.

5 Quinten M, Leitner A, Krenn JR, Aussenegg FR. Electromagnetic energy transport via linear chains of silver nanoparticles. Opt Lett 1998; 23: 1331-1333.
6 Alu A, Engheta N. Effect of small random disorders and imperfections on the performance of arrays of plasmonic nanoparticles. New J Phys 2010; 12: 013015.

7 Liu N, Mukherjee S, Bao K, Li Y, Brown LV et al. Manipulating magnetic plasmon propagation in metallic nanocluster networks. ACS Nano 2012; 6: 5482-5488.

8 Economou EN. Surface plasmons in thin films. Phys Rev 1969; 182: 539-554.

9 Lopez-Tejeira F, Rodrigo SG, Martin-Moreno L, Garcia-Vidal FJ, Devaux E et al. Efficient unidirectional nanoslit couplers for surface plasmons. Nat Phys 2007; 3: 324-328.

10 Bozhevolnyi SI, Volkov VS, Devaux E, Ebbesen TW. Channel plasmon-polariton guiding by subwavelength metal grooves. Phys Rev Lett 2005; 95: 046802.

11 Sanders AW, Routenberg DA, Wiley BJ, Xia YN, Dufresne ER et al. Observation of plasmon propagation, redirection, and fan-out in silver nanowires. Nano Lett 2006; 6: $1822-1826$.

12 Ditlbacher H, Hohenau A, Wagner D, Kreibig U, Rogers M et al. Silver nanowires as surface plasmon resonators. Phys Rev Lett 2005; 95: 257403.

13 Fang YR, Wei H, Hao F, Nordlander P, Xu HX. Remote-excitation surface-enhanced Raman scattering using propagating Ag nanowire plasmons. Nano Lett 2009; 9: 2049-2053.

14 Boardman AD, Aers GC, Teshima R. Retarded edge modes of a parabolic wedge. Phys Rev $B$ 1981; 24: 5703-5712.

15 Burgos SP, Lee HW, Feigenbaum E, Briggs RM, Atwater HA. Synthesis and characterization of plasmonic resonant guided wave networks. Nano Lett 2014; 14: 3284-3292.

16 Huang KC, Seo MK, Sarmiento T, Huo YJ, Harris JS et al. Electrically driven subwavelength optical nanocircuits. Nat Photonics 2014; 8: 244-249.

17 Chen JJ, Sun CW, Hu XY. Nanoscale all-optical devices based on surface plasmon polaritons. Chinese Sci Bull 2014; 59: 2661-2665.

$18 \mathrm{Han} \mathrm{ZH}$, Bozhevolnyi SI. Radiation guiding with surface plasmon polaritons. Rep Prog Phys 2013; 76: 016402.

19 Chang DE, Sorensen AS, Hemmer PR, Lukin MD. Strong coupling of single emitters to surface plasmons. Phys Rev B 2007; 76: 035420.

20 Zhang SP, Wei H, Bao K, Hakanson U, Halas NJ et al. Chiral surface plasmon polaritons on metallic nanowires. Phys Rev Lett 2011; 107: 096801.

21 Schmidt MA, Prill Sempere LN, Tyagi HK, Poulton CG, Russell PS. Waveguiding and plasmon resonances in two-dimensional photonic lattices of gold and silver nanowires. Phys Rev B 2008; 77: 033417.

22 Schmidt MA, Russell PS. Long-range spiralling surface plasmon modes on metallic nanowires. Opt Express 2008; 16: 13617-13623.

23 Zhang SP, Xu HX. Optimizing substrate-mediated plasmon coupling toward highperformance plasmonic nanowire waveguides. ACS Nano 2012; 6: 8128-8135.

24 Moreno E, Garcia-Vidal FJ, Rodrigo SG, Martin-Moreno L, Bozhevolnyi SI. Channel plasmon-polaritons: modal shape, dispersion, and losses. Opt Lett 2006; 31: 34473449.

25 Volkov VS, Bozhevolnyi SI, Rodrigo SG, Martin-Moreno L, Garcia-Vidal FJ et al Nanofocusing with channel plasmon polaritons. Nano Lett 2009; 9: 1278-1282.

26 Bozhevolnyi SI, Volkov VS, Devaux E, Laluet JY, Ebbesen TW. Channel plasmon subwavelength waveguide components including interferometers and ring resonators. Nature 2006; 440: 508-511.

27 Schnell M, Alonso-Gonzalez P, Arzubiaga L, Casanova F, Hueso LE et al. Nanofocusing of mid-infrared energy with tapered transmission lines. Nat Photonics 2011; 5: 283287.

28 Boltasseva A, Volkov VS, Nielsen RB, Moreno E, Rodrigo SG et al. Triangular metal wedges for subwavelength plasmon-polariton guiding at telecom wavelengths. Opt Express 2008; 16: 5252-5260.

29 Johnson JC, Choi HJ, Knutsen KP, Schaller RD, Yang PD et al. Single gallium nitride nanowire lasers. Nat Mater 2002; 1: 106-110.

30 Duan XF, Huang Y, Agarwal R, Lieber CM. Single-nanowire electrically driven lasers. Nature 2003; 421: 241-245.

31 Zimmler MA, Bao J, Capasso F, Muller S, Ronning C. Laser action in nanowires: observation of the transition from amplified spontaneous emission to laser oscillation. Appl Phys Lett 2008; 93: 051101.

32 Oulton RF, Sorger VJ, Zentgraf T, Ma RM, Gladden C et al. Plasmon lasers at deep subwavelength scale. Nature 2009; 461: 629-632.

33 Liu XF, Zhang Q, Yip JN, Xiong QH, Sum TC. Wavelength tunable single nanowire lasers based on surface plasmon polariton enhanced burstein-moss effect. Nano Lett 2013; 13: 5336-5343.

34 Lu YJ, Wang CY, Kim J, Chen HY, Lu MY et al. All-color plasmonic nanolasers with ultralow thresholds: autotuning mechanism for single-mode lasing. Nano Lett 2014; 14: 4381-4388.

35 Ding K, Ning CZ. Metallic subwavelength-cavity semiconductor nanolasers. Light Sci App/2012; 1: e20; doi: 10.1038/lsa.2012.20

36 Lu YJ, Kim J, Chen HY, Wu CH, Dabidian N et al. Plasmonic nanolaser using epitaxially grown silver film. Science 2012; 337: 450-453.

37 Hill MT, Marell M, Leong ES, Smalbrugge B, Zhu YC et al. Lasing in metal-insulatormetal sub-wavelength plasmonic waveguides. Opt Express 2009; 17: 11107 11112 .

38 Ding K, Liu ZC, Yin LJ, Hill MT, Marell MJ et al. Room-temperature continuous wave lasing in deep-subwavelength metallic cavities under electrical injection. Phys Rev $B$ 2012; 85: 041301

39 Lozano G, Louwers DJ, Rodriguez SR, Murai S, Jansen OTA et al. Plasmonics for solidstate lighting: enhanced excitation and directional emission of highly efficient light sources. Light Sci App/ 2013; 2: e66; doi: 10.1038/Isa.2013.22. 
40 Fan PY, Colombo C, Huang KC, Krogstrup P, Nygard J et al. An electrically-driven GaAs nanowire surface plasmon source. Nano Lett 2012; 12: 4943-4947.

41 Walters RJ, van Loon RV, Brunets I, Schmitz J, Polman A. A silicon-based electrica source of surface plasmon polaritons. Nat Mater 2010; 9: 21-25.

42 Shalin AS, Ginzburg P, Belov PA, Kivshar YS, Zayats AV. Nano-opto-mechanical effects in plasmonic waveguides. Laser Photonics Rev 2014; 8: 131-136.

43 Melikyan A, Lindenmann N, Walheim S, Leufke PM, Ulrich S et al. Surface plasmon polariton absorption modulator. Opt Express 2011; 19: 8855-8869.

44 Ma RM, Yin XB, Oulton RF, Sorger VJ, Zhang X. Multiplexed and electrically modulated plasmon laser circuit. Nano Lett 2012; 12: 5396-5402.

45 Melikyan A, Alloatti L, Muslija A, Hillerkuss D, Schindler PC et al. High-speed plasmonic phase modulators. Nat Photonics 2014; 8: 229-233.

46 Alloatti L, Palmer R, Diebold S, Pahl KP, Chen BQ et al. $100 \mathrm{GHz}$ silicon-organic hybrid modulator. Light Sci App/ 2014; 3: e173; doi:10.1038/lsa.2014.54.

47 Gosciniak J, Bozhevolnyi SI. Performance of thermo-optic components based on dielectric-loaded surface plasmon polariton waveguides. Sci Rep 2013; 3: 1803.

48 Yan YL, Zhang C, Zheng JY, Yao JN, Zhao YS. Optical modulation based on direct photon-plasmon coupling in organic/metal nanowire heterojunctions. Adv Mater 2012; 24: 5681-5686.

49 Wei H, Wang ZX, Tian XR, Kall M, Xu HX. Cascaded logic gates in nanophotonic plasmon networks. Nat Commun 2011; 2: 387.

50 Wei H, Li ZP, Tian XR, Wang ZX, Cong FZ et al. Quantum dot-based local field imaging reveals plasmon-based interferometric logic in silver nanowire networks. Nano Let 2011; 11: 471-475.

51 Wu YD, Hsueh YT, Shih TT. Novel All-optical Logic Gates Based on Microring Metalinsulator-metal Plasmonic Waveguides. PIERS Proceedings. EM Academy Stockholm, Sweden; 2013. p.169.

52 Kim NC, Li JB, Yang ZJ, Hao ZH, Wang QQ. Switching of a single propagating plasmon by two quantum dots system. Appl Phys Lett 2010; 97: 061110.

53 Kim NC, Ko MC, Wang QQ. Single plasmon switching with $\mathrm{n}$ quantum dots system coupled to one-dimensional waveguide. Plasmonics 2015; 10: 611-615.

54 Chang DE, Sorensen AS, Demler EA, Lukin MD. A single-photon transistor using nanoscale surface plasmons. Nat Phys 2007; 3: 807-812.

55 Fang YR, Li ZP, Huang YZ, Zhang SP, Nordlander P et al. Branched silver nanowires as controllable plasmon routers. Nano Lett 2010; 10: 1950-1954.

56 Volkov VS, Bozhevolnyi SI, Devaux E, Laluet JY, Ebbesen TW. Wavelength selective nanophotonic components utilizing channel plasmon polaritons. Nano Lett 2007; 7 880-884

57 Wu YD, Huang ML, Chen MH, Tasy RZ. New All-optical Switch Based on the Local Nonlinear Plasmonic Mach-Zehnder Interferometer Waveguides. PIERS Proceedings. EM Academy, Stockholm, Sweden; 2013. p.452

58 Ming TA, Zhao L, Xiao MD, Wang JF. Resonance-coupling-based plasmonic switches. Small 2010; 6: 2514-2519.

59 Li ZP, Zhang SP, Halas NJ, Nordlander P, Xu HX. Coherent modulation of propagating plasmons in silver-nanowire-based structures. Small 2011; 7: 593-596.

60 Guo X, Qiu M, Bao JM, Wiley BJ, Yang Q et al. Direct coupling of plasmonic and photonic nanowires for hybrid nanophotonic components and circuits. Nano Let 2009; 9: 4515-4519.

61 Wang YP, Guo X, Tong LM, Lou JY. Modeling of Au-nanowire waveguide for plasmonic sensing in liquids. J Lightwave Technol 2014; 32: 4233-4238.

62 Liu YK, Wang SC, Li YY, Song LY, Xie XS et al. Efficient color routing with a dispersioncontrolled waveguide array. Light Sci App/2013. 2: e52. doi:10.1038/lsa.2013.8.

63 Chen Z, Holmgaard T, Bozhevolnyi SI, Krasavin AV, Zayats AV et al. Wavelength selective directional coupling with dielectric-loaded plasmonic waveguides. Opt Lett 2009; 34: 310-312.

64 Falk AL, Koppens FH, Yu CL, Kang K, Snapp ND et al. Near-field electrical detection of optical plasmons and single-plasmon sources. Nat Phys 2009; 5: 475-479.
65 Neutens P, Van Dorpe P, De Vlaminck I, Lagae L, Borghs G. Electrical detection of confined gap plasmons in metal-insulator-metal waveguides. Nat Photonics 2009; 3 : 283-286.

66 Goykhman I, Desiatov B, Khurgin J, Shappir J, Levy U. Locally oxidized silicon surfaceplasmon Schottky detector for telecom regime. Nano Lett 2011; 11: 2219-2224.

67 Liu L, Huang L, Hu L. Microcomputer Principle and Interface Technology Chinese Edn. Publishing House of Electronics Industry: Beijing, 2010.

68 Ly-Gagnon DS, Balram KC, White JS, Wahl P, Brongersma ML. Routing and photodetection in subwavelength plasmonic slot waveguides. Nanophotonics-Berlin 2012; 1: 9-16.

69 Lin YM, Dimitrakopoulos C, Jenkins KA, Farmer DB, Chiu HY et al. 100-GHz transistors from wafer-scale epitaxial graphene. Science 2010; 327: 662 .

70 Miller DA. Device requirements for optical interconnects to silicon chips. P IEEE 2009; 97: 1166-1185.

71 Liu N, Wei H, Li J, Wang ZX, Tian XR et al. Plasmonic amplification with ultra-high optical gain at room temperature. Sci Rep 2013; 3: 1967

72 De Leon I, Berini P. Amplification of long-range surface plasmons by a dipolar gain medium. Nat Photonics 2010; 4: 382-387.

73 Wang WH, Yang Q, Fan FR, Xu HX, Wang ZL. Light propagation in curved silver nanowire plasmonic waveguides. Nano Lett 2011; 11: 1603-1608.

74 Volkov VS, Bozhevolnyi SI, Devaux E, Ebbesen TW. Bend loss for channel plasmon polaritons. Appl Phys Lett 2006; 89: 143108

75 Akimov AV, Mukherjee A, Yu CL, Chang DE, Zibrov AS et al. Generation of single optical plasmons in metallic nanowires coupled to quantum dots. Nature 2007; 450: 402-406.

76 Li ZP, Bao K, Fang YR, Huang YZ, Nordlander P et al. Correlation between incident and emission polarization in nanowire surface plasmon waveguides. Nano Lett 2010; 10 : 1831-1835.

77 Li ZP, Shegai T, Haran G, Xu HX. Multiple-particle nanoantennas for enormous enhancement and polarization control of light emission. ACS Nano 2009; 3: 637642.

78 Nagpal P, Lindquist NC, Oh SH, Norris DJ. Ultrasmooth patterned metals for plasmonics and metamaterials. Science 2009; 325: 594-597.

79 Park JH, Nagpal P, McPeak KM, Lindquist NC, Oh SH et al. Fabrication of smooth patterned structures of refractory metals, semiconductors, and oxides via template stripping. Acs Appl Mater Inter 2013; 5: 9701-9708.

80 Dionne JA, Lezec HJ, Atwater HA. Highly confined photon transport in subwavelength metallic slot waveguides. Nano Lett 2006; 6: 1928-1932.

81 Johnson TW, Lapin ZJ, Beams R, Lindquist NC, Rodrigo SG et al. Highly reproducible near-field optical imaging with sub-20-nm resolution based on template-stripped gold pyramids. ACS Nano 2012; 6: 9168-9174.

82 Sard R. Chemical deposition of single crystal gold films. J Electrochem Soc 1970; 117: 1156-1157.

83 Fedotov VA, Uchino T, Ou JY. Low-loss plasmonic metamaterial based on epitaxial gold monocrystal film. Opt Express 2012; 20: 9545-9550.

84 Tame MS, McEnery KR, Ozdemir SK, Lee J, Maier SA et al. Quantum plasmonics. Nat Phys 2013; 9: 329-340.

(c) (i) (2) This work is licensed under a Creative Commons Attribution-

BY NG SA NonCommercial-ShareAlike 3.0 Unported License. The images or other third party material in this article are included in the article's Creative Commons license, unless indicated otherwise in the credit line; if the material is not included under the Creative Commons license, users will need to obtain permission from the license holder to reproduce the material. To view a copy of this license, visit http://creativecommons.org/licenses/by-nc-sa/3.0/ 